\title{
Síndrome de Poland
}

\author{
DEPARTAMENTO DE OBSTETRICIA Y GINECOLOGIA \\ FACULTAD DE MEDICINA DE VALENCIA. HOSPITAL CLINICO \\ UNIVERSITARIO. DIR. PROFESOR BONILLA-MUSOLES \\ UNIVERSIDAD INDUSTRIAL DE SANTANDER - COLOMBIA
}

\author{
Dres.: Millet Part, A; Aviño, J; Blanes* , A. y Luis F. Mendoza**
}

\section{INTRODUCCION}

Dentro del grupo de las anomalías mamarias del desarrollo por defecto o ausencia podemos observar desde la asimetria mamaria, hasta la carencia de este apéndice, tanto en el hombre como en la mujer. Esta falta, que puede ser iatrogénica como consecuencia de maniobras quirúrgicas, radioterápias $\mathrm{o}$ a causa de infecciones antes del desarrollo del botón mamario. También puede ser congénita, como en tres casos que nosotros presentamos.

En uno de ellos, que de entrada excluimos del grupo, había una falta de desarrollo de glandula mamaria con presencia de pezón y areola (músculos pectorales, por lo que lo consideramos como una hipomastia y lo tratamos con la implantación de una protesis inflable subpectoral.

En los otros dos casos, motivo de este trabajo, además de una falta del desarrollo mamario existia una ausencia de pezón y areola así como de los musculos pectorales subyacentes, lo que

\footnotetext{
* Facultad de Medicina de Valencia (España).

* Facultad de Medicina. U.I.S. Bucaramanga
}

conforma los caracteres del Síndrome de Poland.

\section{MATERIAL Y METODO}

Hemos estudiado dos pacientes. La primera es S.H. de veinte años soltera, que acudió por un problema de ataxia menstrual; menarquia a los quince años, con un fenotipo femenino al igual que la pilificación, a excepción de una ligera pilificación en labio superior y barbilla, aparato genital normal. Temperaturas basales alternando ciclos bifásicos y monofásicos siendo la analítica hormonal dentro de los límites de la normalidad.

Al efectuar la exploración general de esta paciente nos encontramos con una ausencia completa de su mama, pezón, areola y musculo pectoral del lado izquierdo siendo normal el desarrollo del lado derecho.

El segundo caso, nos es remitido por los padres de la paciente, va que se trata de una niña de 16 meses de edad, en quien sus padres han notado la falta del pezón del lado derecho. Al examinarla, observamos también, que la niña carece del musculo pectoral de ese lado destacando la parrilla costal por debajo de la piel. El resto de la exploración de la 
PRIMER CASO

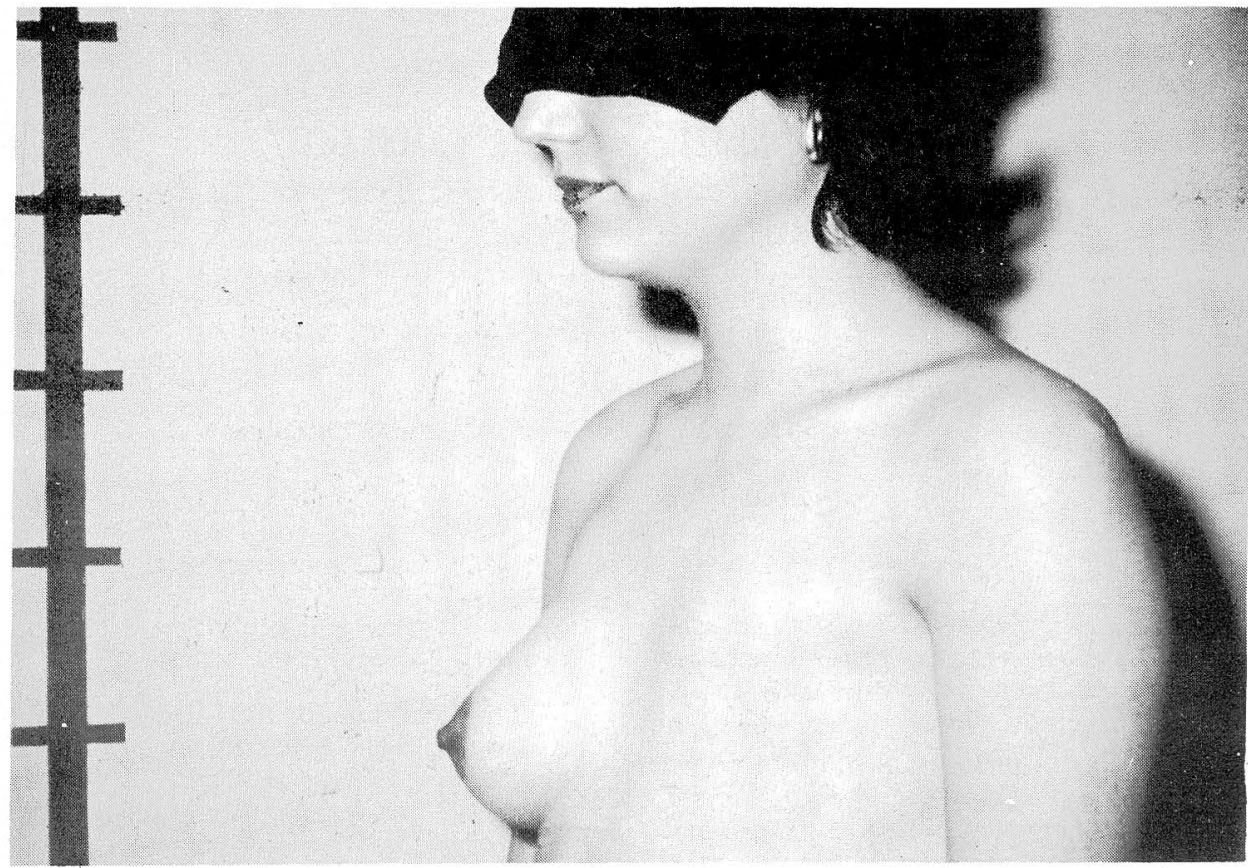

SEGUNDO CASO

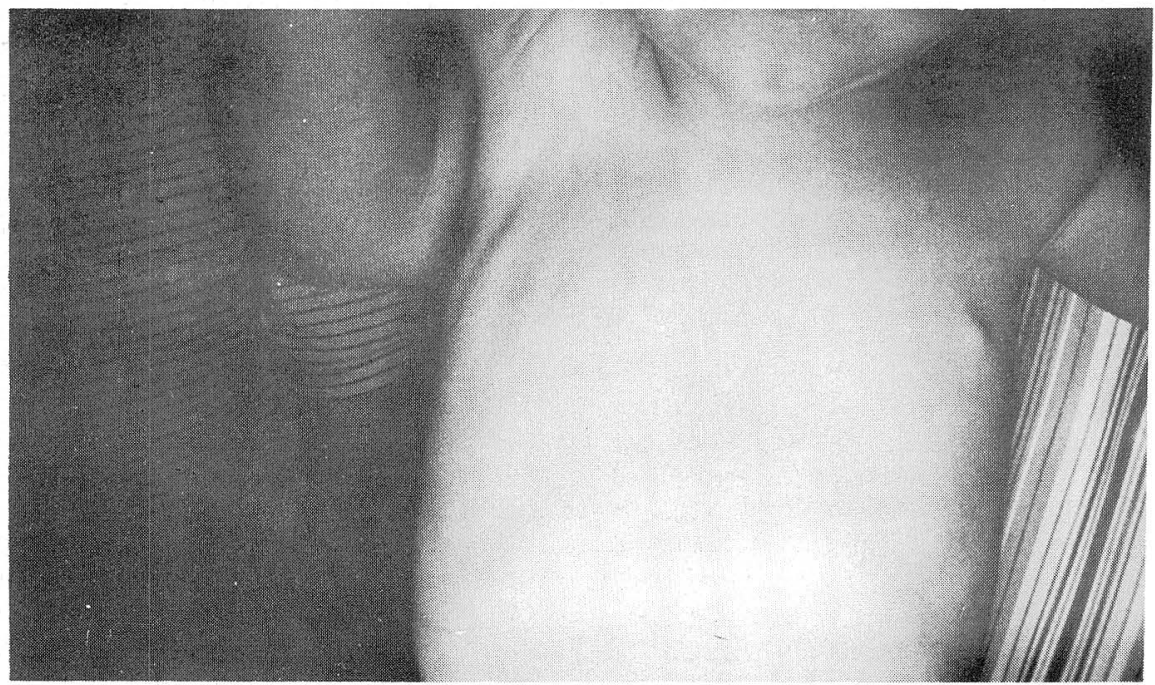


paciente es normal correspondiendo su talla y peso a su edad cronológica y no existiendo patología previa durante su gestación.

Tanto en un caso como en el otro se efectuaron estudios cromosómicos siendo normales $\mathrm{y}$ estudios radiológicos de la caja tóraxica, no observándose alteraciones de las costillas ni del hemicuerpo afectado.

\section{DISCUSION}

Aunque la descripción del Dr. Alfred Poland, del Guy's Hospital en 1841 (5), se refería a un muchacho, es evidente que la agenesia de mama muestra su mayor contrapunto en la mujer, por cuanto este órgano se supone para ella.

Siguen sin conocerse las causas de estos defectos congénitos pero es evidente que es una alteración del embrión de seis a veinte m.m, que es cuando se establece el engrosamiento del éctodermo que dará lugar al pezón y del cual dependerá el progreso mesodermico que dará origen al tejido de sostén (musculos pectorales, costillas y musculos intercostales) e inclusive al esbozo de la extremidad superior de ese lado.

Así pues Poland describió un caso de agenesia de mama unilateral asociada a un musculo pectoral mayor deficiente, con una extremidad superior homolateral más corta que se asociaba a una sindactilia.

El problema no es hereditario, ni familiar ni tiene que ver con ningún estimulo hormonal.

La frecuencia es difícil establecerla por los pocos casos publicados (4) pero estarían dentro del grupo del $3 \%$ al $5 \%$ de tsa pacientes que acuden a someterse a cirugía estética de mama, por hipoplasia unilateral.

(2) Citado por H. Goldwyn
En cuanto al tratamiento, se ha pasado de la inserción de una prótesis premoldeada, según el volumén de la otra mama y con prolongación axilar (ya que al faltarles el pectoral la axila carece esteticamente de pilar anterior) como la ideada por Perras (2), la cual se introducía por vía axilar, teniendo cuidado de fijarla bien, por el peligro de rotación de su porción superior; a la transposición muscular del fascículo proximal del gran dorsal, como si se tratase de una reconstrucción post-mastectomia radical.

Esta se efectua por vía latero-toráxica y posteriormente se efectua la inserción de una prótesis en el plano submuscular. La reconstrucción del pezón será posterior y según las técnicas habituales.

La hipertrofia de la otra mama aparentemente normal es muy frecuente $\mathrm{y} a$ menudo hay que someterla a una mamoplastia de reducción para lograr la mayor simetria posible.

El tratamiento pues queda relegado a la cirugía plástica y no se efectuara antes de los 17-19 años, en que se habrá completado el desarrollo de la otra mama.

\section{RESUMEN}

Se describen dos casos de Síndrome de Poland (ausencia unilateral de mama y musculo pectoral). Su frecuencia es escasa en la clínica de mastología. Se describe la actitud diagnóstica y terapéutica en estas pacientes.

\section{SUMMARY}

We describe two cases of Poland Syndrome (Total absence of one mamal gland and pectoral muscle). Its frequency is poor in the masthology clinic. We describe the actitude diagnostical and the therapeutic in this pacients. 


\section{BIBLIOGRAFIA}

1. FERNANDEZ. CID A. y cols. (1982). Patología mamaria, Salvat Ed.

2. GOLDWYN. R.M. (1981). Cirugía plástica $y$ de reconstrucción de la mama. Salvat Ed.

3. MAILLARD, G. MONTANDON, D. GOIN, J.L. (1983). Chirurgie du Sein Plasties et reconstructions. Masson Ed.
4. MILLER PART, A. Julia, J. (1978). Un caso de amastia unilateral. Toko. Ginecol. Pract. 419. 321-24.

5. POLAND, A. (1841). Deficiency of the pectoral muscles. Guy's Hosp. Clin. Rep. 6, 191 (citado por Goldwyn)

6. URIBURU, J.V. (1977) La mama. López Libreros Ed. Tomo I. 